\title{
Pulmonary arterial hypertension in adult onset Still's disease: a case report of a severe complication
}

\author{
L. Guilleminault ${ }^{1,2,3,5^{*}}$, S. Laurent ${ }^{1}$, A. Foucher ${ }^{4}$, P. Poubeau ${ }^{4}$ and F. Paganin ${ }^{1,3}$
}

\begin{abstract}
Background: Adult onset of Still's disease (AOSD) is a rare systemic inflammatory disease. Cardiorespiratory complications are mainly represented by pleural and pericardial disorders and are less frequent than cutaneous and articular complaints. Pulmonary arterial hypertension (PAH) occurring in AOSD is rarely described in literature.

Case presentation: We present the case of a young patient who developed severe PAH 2 years after diagnosis of AOSD. This is a rare and severe complication which is probably underestimated.

Conclusions: PAH in AOSD can be lethal, and unfortunately its occurrence is unpredictable. Echocardiographic screening of AOSD patients should be evaluated in further trials. Currently, the most suitable treatment is still unknown.
\end{abstract}

Keywords: Case report, Pulmonary arterial hypertension, Adult onset Still's disease, Auto-inflammatory disease

\section{Background}

Adult onset Still's disease (AODS) is a rare autoinflammatory disease presenting with a wide range of non-specific symptoms [1]. In most cases, patients have spiking fever, arthralgia, rash and high leukocytosis, which are major items of criteria described in the literature [2,3]. Cardio-respiratory complications are mainly represented by pleural and pericardial disorders $[4,5]$. We report herein the case of a young woman with AOSD who developed pulmonary hypertension. This complication is rarely observed but pathophysiology of this phenomenon is unkown.

\section{Case presentation}

Initial presentation and diagnosis

A 19 year-old female presented with persistent fever, evanescent rash (Fig. 1), sore throat and polyarthralgia without synovitis (bilateral knees, shoulders, elbows and metatarsophalangeal joints). Physical examination showed axillary and inguinal lymphadenopathy (diameter <

\footnotetext{
* Correspondence: laurent.guilleminault@chu-reunion.fr

${ }^{1}$ Service de Pneumologie, CHU Reunion/GHSR, Saint-Pierre F-97410, France ${ }^{2}$ Inserm, UMR 1188 Diabète athérothrombose Thérapies Réunion Océan Indien (DéTROI), plateforme CYROI, Sainte-Clotilde F-97490, France Full list of author information is available at the end of the article
}

$1 \mathrm{~cm})$. Investigations revealed a high erythrocyte sedimentation rate $(87 \mathrm{~mm} /$ first hour) and high C-reactive protein (CRP) $(116 \mathrm{mg} / \mathrm{L})$. Serum creatinine, liver enzymes, CPK, granulocytes and platelet levels were all normal. Rheumatoid factor, anti CCP, antinuclear antibodies and antineutrophil cytoplasm antibodies were negative. Blood cultures were negative. Serological tests for virus (EBV, CMV, HSV and HIV) were negative. Ferritin level was high $(8140 \mu \mathrm{g} / \mathrm{L})$ and glycosylated ferritin was low (rate: $9 \%$ ). Computed tomography (CT) scan of chest, abdomen and pelvis was normal (Fig. 2a). The presentation suggested a systemic rheumatic disease and the investigations excluded alternative auto-immune diagnoses. AOSD was finally proposed, as a diagnosis of exclusion, based on the association of arthritis, rash, fever, elevated acute phase reactants and low glycosylated ferritin.

\section{Treatment}

She received oral steroids $(1 \mathrm{mg} / \mathrm{kg})$, which led to clinical and laboratory improvement. Symptoms reappeared during the tapering-off phase $\left(25 \mathrm{mg} \cdot \mathrm{d}^{-1}\right)$ and the dose was increased again to $1 \mathrm{mg} / \mathrm{kg}$ with the addition of a $10 \mathrm{mg}$ methotrexate dose once a week. Despite methotrexate therapy, the patient experienced a second relapse when the steroid dose was decreased $\left(25 \mathrm{mg} \cdot \mathrm{d}^{-1}\right)$. 


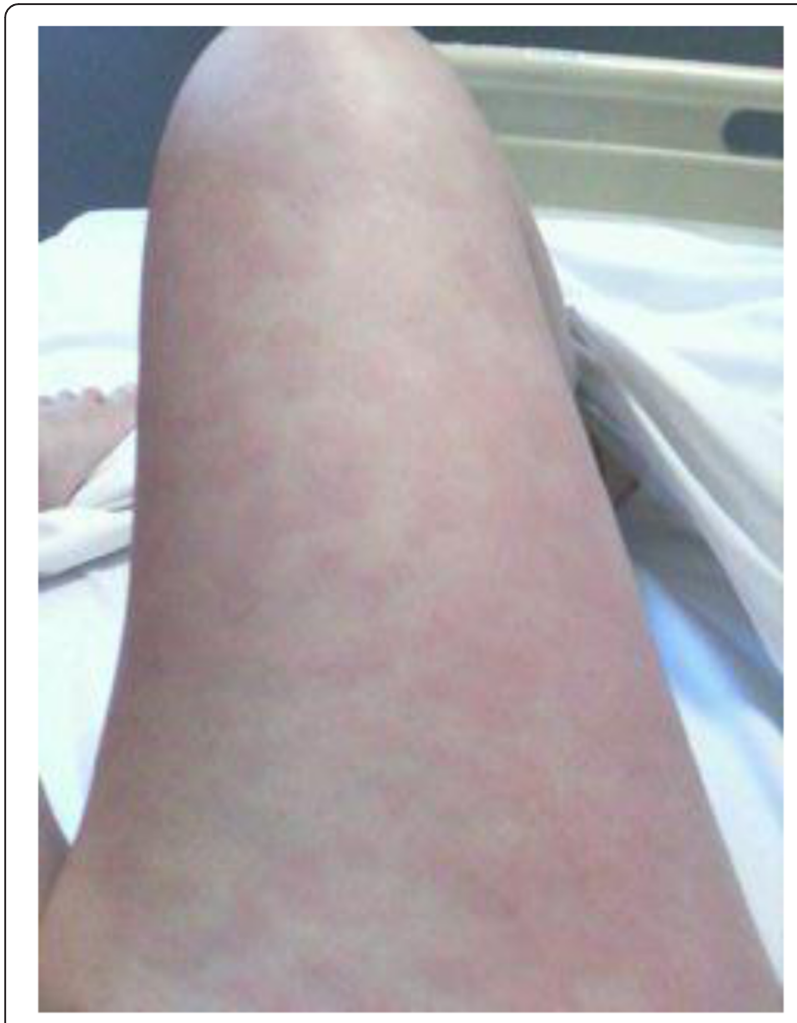

Fig. 1 Evanescent urticaria-like rash on right leg in our patient with adult-onset Still's disease

Methotrexate dose was increased to $15 \mathrm{mg}$ once per week, with a steroid dose at $25 \mathrm{mg} \cdot \mathrm{d}^{-1}$. Decision was made to introduce anakinra $\left(100 \mathrm{mg} \cdot \mathrm{d}^{-1}\right)$ as a steroid sparing agent. Steroid dosage was decreased and methotrexate was discontinued but subsequently reintroduced due to a reappearance of skin rash.

\section{Respiratory complication}

Two years after AOSD diagnosis, the patient presented with NYHA class III dyspnea associated with polyarthritis, fatigue and ferritin increase. At that time, therapy was methotrexate (15 mg once per week), corticosteroids (25 mg.d ${ }^{-1}$ ) and anakinra. There was no sign of right- sided heart failure and the electrocardiogram was normal. The patient walked $462 \mathrm{~m}$ with oxygen desaturation (SpO2 : $87 \%)$ on a 6 -min walk test (6MWT). Vital capacity was $1.6 \mathrm{~L}$ (49\%), and total pulmonary capacity 2.44 L (59 \%). Carbon monoxide diffusion capacity was low (22\%). We did not have any reference values as the patient had no previous pulmonary function tests available. Arterial blood gas analysis revealed hypoxemia (pO2 : $71 \mathrm{~mm} \cdot \mathrm{Hg}^{-1}$ ) and hypocapnia (pCO2 : $34 \mathrm{~mm} . \mathrm{Hg}$ $\left.{ }^{-1}\right)$. BNP, troponin, serum uric acid, creatinin and CRP were normal. Chest HRCT showed a dilated pulmonary trunk (PT) (31 mm); the PT/Aorta trunk index was 1.4 and the right ventricle/left ventricle $(\mathrm{RV} / \mathrm{LV})$ index was 1.26 (Fig. 2b). There was no pleural or parenchymal involvement of the lungs; no signs of pulmonary vasculitis, and no pulmonary embolism as confirmed by a ventilation/perfusion scan. Doppler echocardiography showed a systolic pulmonary artery pressure of $65 \mathrm{~mm} \cdot \mathrm{Hg}^{-1}$. Pulmonary hypertension was confirmed by right heart catheterization with a mean pulmonary artery pressure (mPAP) of $33 \mathrm{~mm} \cdot \mathrm{Hg}^{-1}$ and normal PCWP $\left(4 \mathrm{~mm} \cdot \mathrm{Hg}^{-1}\right)$. The cardiac output was 4.0 L.mn ${ }^{-1}$ and pulmonary resistance was 7.25 UWood. A Nitric oxide test was negative.

\section{Outcome}

The patient was included in a clinical trial and received ambrisentan and tadalafil associated with ongoing anakinra and corticosteroids. Methotrexate was removed to avoid adverse effects on the lungs or liver. Ambrisentan was rapidly discontinued due to cytolytic hepatitis. One month later, she was admitted to ICU for right-sided heart failure; a thrombotic thrombocytopenic purpura was observed (low platelets at $5 \mathrm{G} / \mathrm{L}$, anaemia at $6.7 \mathrm{~g} / \mathrm{dL}$, LDH: 905 UI/L ADAMTS13: 31 \% with N: 50-150\%). Dobutamine and intraveinous (IV) prostacycline were administered, with a progressive improvement. The patient showed a clinical improvement with a distance of $696 \mathrm{~m}$ on 6MWT under IV protacycline, tadalafil, anakinra and corticosteroids. One month later, she was admitted again to intensive care for septic shock with a staphylococcus epidermidis infection. The tunneled catheter was removed
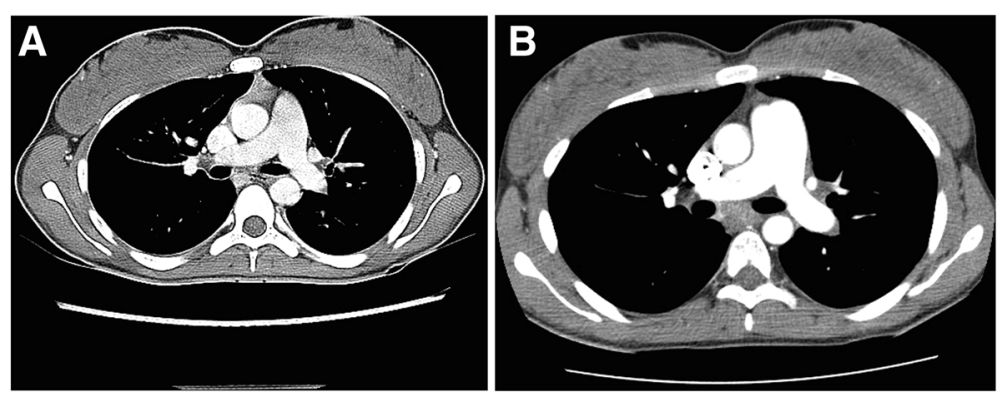

Fig. 2 a CT scan performed at AOSD diagnosis with a pulmonary trunk (PT) at $21.7 \mathrm{~mm}$, aorta trunk at $22.8 \mathrm{~mm}$. PT/Aorta trunk index was 0.95 . b CT scan performed 2 years after diagnosis with a pulmonary trunk (PT) at $31 \mathrm{~mm}$, aorta trunk at $22.1 \mathrm{~mm}$. PT/Aorta trunk index was 1.4 
and bacteriological analyses found staphylococcus epidermidis. Evolution was poor and she passed away despite antibiotic treatment (piperacillin/amikacin) and vasoactive drug support.

\section{Discussion}

AOSD is a rare systemic autoinflammatory disease with a prevalence ranging from 1 to 34 cases per million people [6-8]. Young people are preferentially affected with a mean age at diagnosis around 36 years old $[9,10]$. Clinical presentation is not specific and a wide range of symptoms may be observed [4]. A high white blood cell count is common but was not observed in our patient. No laboratory test is specific but a low glycosylated ferritin $(<20 \%)$ may be suggestive.

In a ten-year retrospective study, none of the $28 \mathrm{pa}-$ tients with an AOSD diagnosis died [11]. The median follow-up was 3.72 years. However, other studies report a mortality rate around $10 \%[12,13]$. Life expectancy and quality of life in AOSD patients may deteriorate with some complications. Reactive hemophagocytic lymphohistiocytosis seems to be the most frequent complication (about 12-15\%) and is particularly associated with a high mortality rate [14]. Other complications such as pericarditis, pleuritis, disseminated intravascular coagulation, thrombotic microangiopathy, fulminant hepatitis and AA amyloidosis are rarely observed [15-19].

$\mathrm{PAH}$ is a very rare complication of AOSD [1]. Its prevalence is probably under-estimated as only symptomatic case reports have been documented. Only 9 cases of PAH in AOSD patients have been published in the literature (Additional file 1: Table S1) [20-27]. Age of onset varies from 18 to 38 years old. A female predominance was reported ( 8 females to 1 male). Onset of PAH ranges from 0 to 108 months after AOSD diagnosis with a median of 24 months, which is concordant with our case. PAH may be a life-threatening complication with a $40 \%$ fatality in previously reported cases. However, it is possible that authors preferentially published fatal cases, therefore introducing a bias in the prognosis.

Pathophysiology of PAH with AOSD is unknown. AOSD is an autoinflammatory disease in which pathogenesis is dominated by neutrophil and macrophage (innate immunity cells) activation. Several non-specific cytokines and chemokines such as IL-1 $\beta$, IL-6, IL-18 and TNF $\alpha$ are particularly involved in AOSD pathogenesis [28-30]. In idiopathic PAH (IPAH) or connective tissue disease associated with PAH (CTD-PAH), it is widely accepted that alteration of immune mechanisms plays a significant role by recruiting inflammatory cells, remodeling the pulmonary vasculature, and promoting autoimmune responses [31]. IL-1 $\beta$, IL-6, IL-18 and other cytokines are increased in IPAH patients, reflecting a dysregulation of innate immunity, also observed in AOSD. Pulmonary microangiopathy, which is similar to renal involvement and thrombotic thrombocytopenic purpura (which was observed in our patient), could participate in pathogenesis of PAH with AOSD [25]. Further studies are needed to properly characterise the pathogenesis in order to classify PAH with AOSD in the Pulmonary Hypertension World Health Organisation (WHO) clinical classification system. Similarities with IPAH and CTD could lead to classification of AOSD $\mathrm{PAH}$ as group 1.

In systemic sclerosis, the high incidence and prevalence of PAH associated with a devastating evolution in untreated cases, led to the recommendation of systematic and regular echocardiography screening in this high risk population. In our opinion, echocardiography screening in AOSD could have a major impact: (1) to determine PAH prevalence in AOSD patients. (2) to evaluate real prognosis and specific mortality. (3) to include these patients in PAH treatment clinical trials.

Considering the major role of IL-1 in AOSD, Anakinra was used in several case reports of AOSD patients with $\mathrm{PAH}$ because of its favourable systemic and rheumatic effect. However, in previous case reports, 3 out of 4 patients treated with anakinra worsened. Vasodilator therapies could be an option but have to be evaluated. To our knowledge, no data are available about vasodilator effect in PAH with AOSD. Manipulation of a tunnelled catheter in a patient treated with immunosuppressant drugs increases the risk of infection and prostacycline use should be discussed. Only one case of PAH improvement after tocilizumab, an anti-IL- 6 antibody, has been reported in the literature. IL-6 plays a key role in the pathophysiology of AOSD but also in PAH. Tocilizumab may be an option in this indication.

\section{Conclusion}

In conclusion, $\mathrm{PAH}$ in AOSD is a rare but potentially life-threatening complication. Pathogenesis is unknown but similarities between AOSD and IPAH or CTD-PAH on innate immunity dysregulation may explain a part of the pathophysiology. Screening AOSD patients with echocardiography should be assessed to determine the impact of PAH. Anakinra is used as first line therapy after induction with corticosteroids. Tocilizumab may be used as a second line therapy. To the best of our knowledge, vasodilators have never been assessed in this indication. Clinical trials about biologics or vasodilator treatments are mandatory.

\section{Ethics of approval}

Not applicable. 


\section{Consent}

Written informed consent was obtained from the permitted assigns for publication of this case report and any accompanying images. A copy of the written consent is available for review by the Editor of this journal.

\section{Availability of data and materials \\ Data are not available for obvious reasons of patient confidentiality.}

\section{Additional file}

Additional file 1: Table S1. Case reports of pulmonary arterial

hypertension in adult onset Still's disease in the literature. (DOC 35 kb)

\begin{abstract}
Abbreviations
6MWT: 6-min walk test; AOSD: Adult onset Still's disease; CRP: c-reactive protein; CT scan: computed tomography scan; CTD-PAH: connective tissue disease associated with pulmonary hypertension; IL-1: interleukin-1; IPAH: idiopathic pulmonary arterial hypertension; mPAP: mean pulmonary artery pressure; PAH: pulmonary arterial hypertension.
\end{abstract}

\section{Competing interests}

The authors declare that they have no competing interests.

\section{Authors' contributions}

$L G$ has been involved in drafting the article and has given final approval of the version to be published. SL has been involved in drafting the article. AF and PP have been involved in the management of the patient and have made contributions to acquisition of data. FP has given final approval of the version to be published. All authors have read and approved the final manuscript.

\section{Authors' information}

Not applicable.

\section{Aknowledgement}

Not applicable.

\section{Funding}

No source of funding.

\section{Author details}

${ }^{1}$ Service de Pneumologie, CHU Reunion/GHSR, Saint-Pierre F-97410, France. ${ }^{2}$ Inserm, UMR 1188 Diabète athérothrombose Thérapies Réunion Océan Indien (DéTROI), plateforme CYROI, Sainte-Clotilde F-97490, France. ${ }^{3}$ Université de La Réunion, UMR 1188, Sainte-Clotilde F-97490, France. ${ }^{4}$ Service de maladies infectieuses, CHU Reunion/GHSR, Saint-Pierre F-97410, France. ${ }^{5}$ Service de Pneumologie, Groupe Hospitalier Sud Réunion, BP 350 , 97448 Saint-Pierre cedex, France.

Received: 15 February 2016 Accepted: 1 May 2016 Published online: 10 May 2016

\section{References}

1. Kadavath S, Efthimiou P. Adult-onset Still's disease-pathogenesis, clinical manifestations, and new treatment options. Ann Med. 2015:47:6-14.

2. Yamaguchi M, Ohta A, Tsunematsu T, Kasukawa R, Mizushima Y, Kashiwagi H, Kashiwazaki S, Tanimoto K, Matsumoto Y, Ota T. Preliminary criteria for classification of adult Still's disease. J Rheumatol. 1992:19:424-30.

3. Fautrel B, Zing E, Golmard J-L, Le Moel G, Bissery A, Rioux C, Rozenberg S, Piette J-C, Bourgeois P. Proposal for a new set of classification criteria for adult-onset still disease. Medicine (Baltimore). 2002;81:194-200.

4. Ohta A, Yamaguchi M, Kaneoka H, Nagayoshi T, Hiida M. Adult Still's disease: review of 228 cases from the literature. J Rheumatol. 1987;14:1139-46.

5. Cheema GS, Quismorio FP. Pulmonary involvement in adult-onset Still's disease. Curr Opin Pulm Med. 1999;5:305-9.
6. Magadur-Joly G, Billaud E, Barrier JH, Pennec YL, Masson C, Renou P, Prost A. Epidemiology of adult Still's disease: estimate of the incidence by retrospective study in west France. Ann Rheum Dis. 1995;54:587-90.

7. Wakai K, Ohta A, Tamakoshi A, Ohno Y, Kawamura T, Aoki R, Kojima M, Lin Y, Hashimoto S, Inaba Y, Minowa M, Aizawa S, Ichikawa Y, Miyasaka N. Estimated prevalence and incidence of adult Still's disease: findings by a nationwide epidemiological survey in Japan. J Epidemiol. 1997;7:221-5.

8. Evensen KJ, Nossent HC. Epidemiology and outcome of adult-onset Still's disease in Northern Norway. Scand J Rheumatol. 2006;35:48-51.

9. Gerfaud-Valentin M, Maucort-Boulch D, Hot A, Iwaz J, Ninet J, Durieu I, Broussolle C, Sève $P$. Adult-onset still disease: manifestations, treatment, outcome, and prognostic factors in 57 patients. Medicine (Baltimore). 2014;93:91-9.

10. Colina M, Zucchini W, Ciancio G, Orzincolo C, Trotta F, Govoni M. The evolution of adult-onset Still disease: an observational and comparative study in a cohort of 76 Italian patients. Semin Arthritis Rheum. 2011:41:279-85.

11. Uppal SS, Al-Mutairi M, Hayat S, Abraham M, Malaviya A. Ten years of clinical experience with adult onset Still's disease: is the outcome improving? Clin Rheumatol. 2007;26:1055-60.

12. Zeng T, Zou Y-Q, Wu M-F, Yang C-D. Clinical features and prognosis of adultonset still's disease: 61 cases from China. J Rheumatol. 2009:36:1026-31.

13. Kim H-A, Sung J-M, Suh C-H. Therapeutic responses and prognosis in adultonset Still's disease. Rheumatol Int. 2012:32:1291-8.

14. Gerfaud-Valentin M, Jamilloux Y, Iwaz J, Sève P. Adult-onset Still's disease. Autoimmun Rev. 2014:13:708-22.

15. Masson C, Le Loët X, Lioté F, Renou P, Dubost JJ, Boissier MC, Brithmer L, Brégeon C, Audran M. Adult Still's disease: part I. Manifestations and complications in sixty-five cases in France. Rev Rhum Engl Ed. 1995;62:748-57.

16. Yokoyama M, Suwa A, Shinozawa T, Fujii T, Mimori T, Akizuki M, Ichikawa Y, Tojo T. A case of adult onset Still's disease complicated with adult respiratory distress syndrome and disseminated intravascular coagulation. Nihon Rinsho Meneki Gakkai Kaish. 1995:18:207-14. Abstract.

17. Salamon L, Salamon T, Morovic-Vergles J. Thrombotic microangiopathy in adult-onset Still's disease: case report and review of the literature. Wien Klin Wochenschr. 2009;121:583-8

18. Dino O, Provenzano G, Giannuoli G, Sciarrino E, Pouyet M, Pagliaro L. Fulminant hepatic failure in adult onset Still's disease. J Rheumatol. 1996;23:784-5.

19. Quéméneur $T$, Noel L-H, Kyndt $X$, Droz D, Fleury D, Binaut R, Lemaitre V, Gobert P, Vanhille P. Thrombotic microangiopathy in adult Still's disease. Scand J Rheumatol. 2005:34:399-403.

20. Zen A, Yamashita N, Ueda M, Asakawa Y, Yoshikawa Y, Funai T, Hashimoto T. A case of adult Still's disease with pulmonary hypertension. Ryumachi. 1990:30:45-52. Abstrac

21. Chen $\mathrm{C}-\mathrm{H}$, Chen H-A, Wang H-P, Liao H-T, Chou C-T, Huang D-F. Pulmonary arterial hypertension in autoimmune diseases: an analysis of 19 cases from a medical center in northern Taiwan. J Microbiol Immunol Infect. 2006;39:162-8.

22. Mubashir E, Ahmed MM, Hayat S, Heldmann M, Berney SM. Pulmonary hypertension in a patient with adult-onset Stills disease. Clin Rheumatol. 2007:26:1359-61.

23. Campos M, Schiopu E. Pulmonary arterial hypertension in adult-onset Still's disease: rapid response to anakinra. Case Rep Rheumatol. 2012:2012:537613.

24. de Siqueira ME M, Rodrigues RP, Kormann APM. Adult-onset Still's disease and pulmonary arterial hypertension. Respiratory Med CME. 2009;2:70-2.

25. Thakare M, Habibi S, Agrawal S, Narsimulu G. Pulmonary arterial hypertension complicating adult-onset Still's disease. Clin Rheumatol. 2013;32 Suppl 1:S1-2.

26. Robillard N, Wistaff R, Lamarre-Cliche M, Nguyen PV, Laskine M. Still out of breath? J Med Cases. 2013;4:340-4

27. Kadavath S, Zapantis E, Zolty R, Efthimiou P. A novel therapeutic approach in pulmonary arterial hypertension as a complication of adult-onset Still's disease: targeting IL-6. Int J Rheum Dis. 2014:17:336-40.

28. Kastner DL, Aksentijevich I, Goldbach-Mansky R. Autoinflammatory disease reloaded: a clinical perspective. Cell. 2010;140:784-90.

29. Carroll HP, Paunovic V, Gadina M. Signalling, inflammation and arthritis: crossed signals: the role of interleukin-15 and -18 in autoimmunity. Rheumatology (Oxford). 2008:47:1269-77.

30. Choi J-H, Suh C-H, Lee Y-M, Suh Y-J, Lee S-K, Kim S-S, Nahm D-H, Park H-S Serum cytokine profiles in patients with adult onset Still's disease. J Rheumatol. 2003;30:2422-7.

31. Huertas A, Perros F, Tu L, Cohen-Kaminsky S, Montani D, Dorfmüller P, Guignabert C, Humbert M. Immune dysregulation and endothelial dysfunction in pulmonary arterial hypertension: a complex interplay. Circulation. 2014;129:1332-40. 